\title{
Influence of shock waves on structural and morphological properties of copper oxide NPs for aerospace applications
}

\author{
A. Rita $^{1} \cdot$ A. Sivakumar ${ }^{1}$ - S. A. Martin Britto Dhas ${ }^{1}$
}

Received: 11 June 2019 / Accepted: 29 July 2019 / Published online: 8 August 2019

(c) The Author(s) 2019

\begin{abstract}
The present work is focused on stability of shock wave-exposed copper oxide $(\mathrm{CuO})$ nanoparticles. $\mathrm{CuO}$ nanoparticles are synthesized by chemical reduction method and exposed to 100 shock pulses having Mach number 2.4. The table top semiautomatic pressure-driven shock tube is used to generate shock waves for the present experiment. The influence of shock waves on the treated and untreated $\mathrm{CuO}$ nanoparticles are explored and characterized by a variety of properties like structural, molecular and morphological details observed using powder XRD, FTIR and SEM, respectively. The powder XRD profile confirmed that there are no lattice defects or any deformation except negligible changes in grain size. SEM images established that the shock wave-loaded $\mathrm{CuO}$ nanoparticles have good structural and morphological stability. The obtained results showed that $\mathrm{CuO}$ nanoparticles can be used in aerospace, nuclear reactors and high-pressure applications which undergo extreme conditions. The details are presented intensely in the following sections.
\end{abstract}

\section{Graphic abstract}
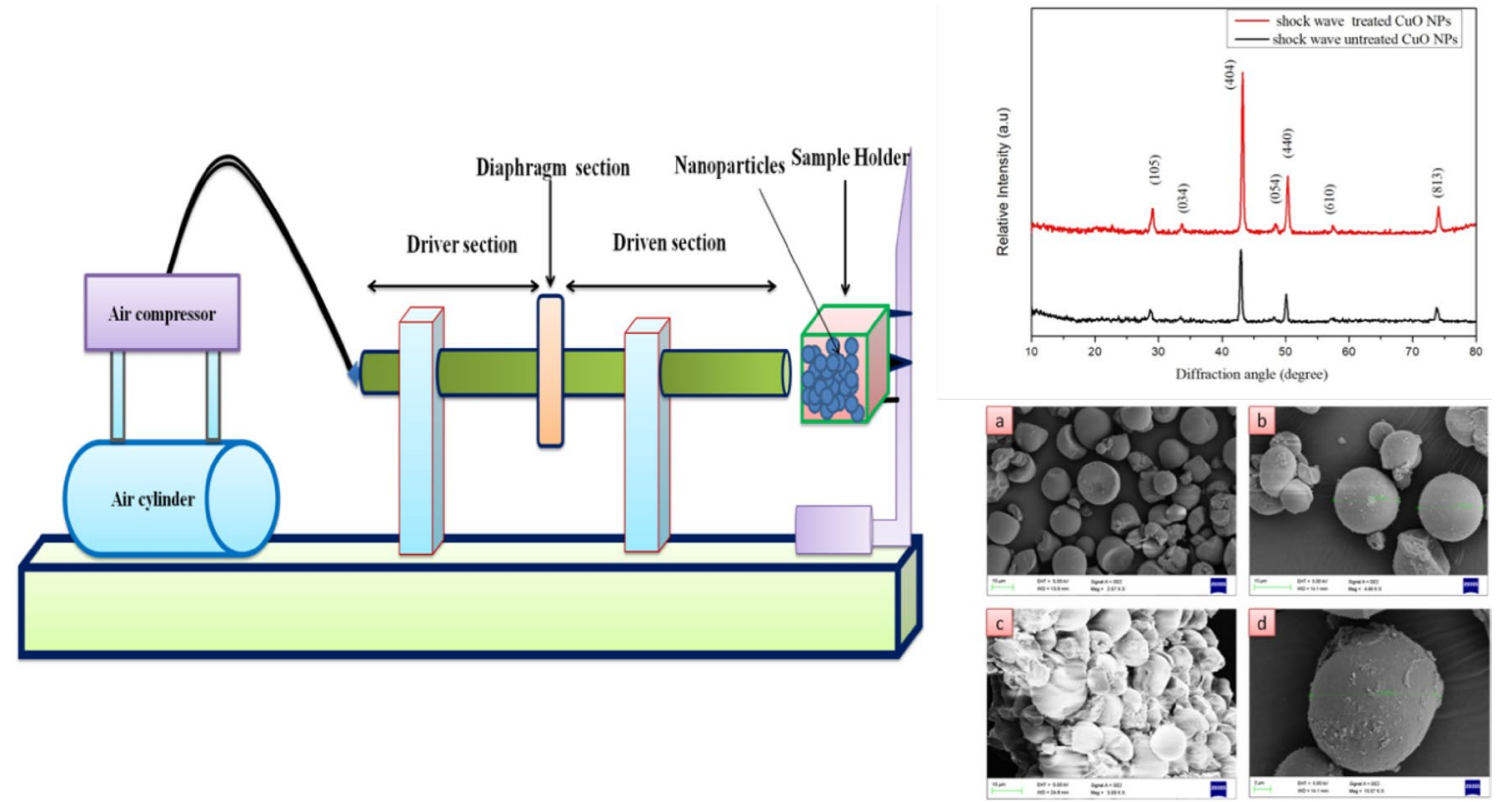

Keywords $\mathrm{CuO}$ nanoparticles $\cdot$ Shock wave $\cdot$ Shock tube $\cdot$ Structural stability $\cdot$ Grain size

\section{S. A. Martin Britto Dhas}

martinbritto@shctpt.edu

1 Department of Physics, Abraham Panampara Research

Centre, Sacred Heart College (Autonomous),

Tirupattur, Vellore, Tamilnadu 635601, India 


\section{Introduction}

In recent years, metal oxide nanoparticles (NPs) like copper oxide, titanium oxide, zinc oxide, etc., are most abundantly used in numerous potential applications, viz., catalysts, solar cell technology, batteries, drug delivery, anti bacterial activity and electronic devices due to their spectacular structural and optical properties [1,2]. Copper oxide is a $P$-type semiconductor material which can be effortlessly obtained by the oxidation of $\mathrm{Cu}$ and extensively used for many applications $[3,4]$. Though metal oxides have very good physical properties in the ambient condition, the mechanical stability and structural stability of the materials are uncertain when they are used in aerospace and nuclear reactor which undergo extreme environmental conditions such as high dynamic pressure and temperature. Thus, the investigations on properties of materials under intense circumstances are very much essential for the device fabrication of such applications [5]. Shock wave is a nonlinear high-pressure phenomena and it has high dynamic pressure and temperature. Producing this sort of shock waves is possible in the laboratory by using shock tubes. When materials are exposed to shock waves, it provides uniaxial stress and makes significant changes in the material properties. The changes occurred in the materials are mainly associated with the structural and crystalline nature of the material. In the last decade, shock wave recovery experiments get significant attractions in material science and structural engineering applications. Shock wave recovery study is very simple and efficient method to examine the material properties in extreme conditions such as dynamic high pressure and temperature. Many of the recent publications show the significant importance of the shock wave recovery experiments in material science branch especially in nano-sized particles [6-8]. To show the significance of shock recovery experiments, few examples of recent reports are listed below. Brontvein et al. [9] utilized shock waves as a growth promoter to synthesize two-step $\mathrm{MoS}_{2}$ nano-tubes with argon as driver gas and phase transformation was demonstrated in fluorite $\mathrm{CeO}_{5} \mathrm{CrO} \cdot 5 \mathrm{O}_{2+x}$ under shock wave-loaded conditions [10]. Cubic zirconia showed unstable electronic structure and crystalline structure and $\mathrm{CeO}_{2}$ showed the stable physical properties in shock wave-loaded conditions $[11,12]$. In shock wave-loaded conditions, lot of pores and cracks were developed in diverse sizes on the surface of $\mathrm{CeO}_{2}$ and the formation of $\mathrm{CeO}_{2-x} \mathrm{~N}_{x}$ are confirmed when strong shock waves interacted with $\mathrm{CeO}_{2}$ ceramic material in the presence of $\mathrm{O}_{2}$ and $\mathrm{N}_{2}$ gases [12]. Nano-crystalline $\mathrm{SiC}$ ceramics showed a distinct change on elastic nature and also transitional plastic structural phase transformation was observed while plane shock wave loaded on it
[13]. $\mathrm{Cu}$ foams with open cell nanoporus showed remarkable effects of relative mass density and specific surface area by the expose of temperature shock [14]. High shock absorptions and negligible changes in density distribution of particles were observed in silica aerogel under shock compressions [15]. The overview of literature studies showed that shock waves can alter the structural, electrical, morphological and optical properties of materials. The present investigation is aimed to understand the stability of the structure and morphology of prepared $\mathrm{CuO}$ NPs under shock wave-exposed conditions. The interesting aspects of the behaviour of shock wave treated and untreated $\mathrm{CuO}$ NPs are discussed in the following sections.

\section{Experimental section}

\section{Synthesis of CuO NPs}

$\mathrm{CuO}$ nanoparticles are synthesized by chemical reduction method by using copper (II) sulphate pentahydrate as precursor salt and starch as capping agent. A calculated amount of starch $(1.2 \mathrm{~g})$ is dissolved in $120 \mathrm{ml}$ of double distilled water under the vigorous stirring for $2 \mathrm{~h}$. Then $0.1 \mathrm{~mol}$ of copper (II) sulphate penta hydrate as a precursor salt is added in $20 \mathrm{ml}$ of water. Then the dissolved precursor solution is slowly added drop by drop into the dissolved starch solution with the constant stirring. Now, the colour of the solution becomes light blue. In the second step, $0.2 \mathrm{~mol}$ of ascorbic acid is added into the prepared solution. While ascorbic acid is added drop by drop, the colour of the solution turned into light green colour and this solution is stirred for $5 \mathrm{~min}$. Simultaneously, $0.5 \mathrm{~mol}$ of sodium hydroxide aqueous solution is slowly added with continuous stirring and the light green colour turned into ochre colour. The prepared solution is heated at $60{ }^{\circ} \mathrm{C}$ for $1 \mathrm{~h}$ and allowed to settle for $24 \mathrm{~h}$ until brown colour precipitate is obtained. The solution was filtered and the precipitate was washed with deionized water and ethanol and dried at room temperature [16]. The synthesized copper oxide nanoparticles are treated with shock wave. The reaction scheme is as shown below:

$$
\begin{aligned}
& \mathrm{CuSO}_{4} \cdot 5 \mathrm{H}_{2} \mathrm{O}+2 \mathrm{NaOH} \rightarrow \mathrm{Na}_{2} \mathrm{SO}_{4}+\mathrm{Cu}(\mathrm{OH})_{2}+5 \mathrm{H}_{2} \mathrm{O} \\
& \mathrm{Cu}(\mathrm{OH})_{2} \rightarrow \mathrm{CuO}+\mathrm{H}_{2} \mathrm{O}
\end{aligned}
$$

\section{Loading of shock waves}

An indigenously fabricated table top semiautomatic shock tube which can generate shock waves from Mach number 1 to 4.5 is used to generate shock waves. This pressure-driven shock tube consists of five sections such as input reservoir, driver, driven, diaphragm and sensor sections, respectively. Input section is used to supply the pressure and the driven 
section is maintained at room pressure. Diaphragm section connects both driver and driven sections. The pressure of the driver section is increased by the compressor and at critical stage the diaphragm ruptures and the shock wave formed into the driven section and move towards the sensor section. Then synthesized copper oxide nanoparticles were exposed to 100 shock pulses with 2.4 Mach number. Shock pulses with Mach number 2.4 having the pressure $\left(P_{5}\right)$ of $2.683 \mathrm{MPa}$ and temperature $\left(T_{5}\right)$ of $989 \mathrm{~K}$ was obtained using 180 GSM carbonless paper diaphragms. The schematic diagram of the experimental setup of shock loading is shown in the Fig. 1. The design and calibration of shock tube performance is communicated in Elsevier [19].

\section{Results and discussion}

\section{FTIR spectroscopic analysis}

The composition and modes of vibration of $\mathrm{CuO}$ nanoparticles were analysed by FTIR spectrometer (Perkin Elmer-50) in the range between 400 and $4000 \mathrm{~cm}^{-1}$. The obtained FTIR spectra are shown in Fig. 2, which confirms the formation of copper oxide nanoparticles with the ascorbic acid as a capping agent. The sharp peaks occurred at $513 \mathrm{~cm}^{-1}$ and $622 \mathrm{~cm}^{-1}$ indicate the presences of $\mathrm{Cu}-\mathrm{O}$ stretching. The peak arising at $1019 \mathrm{~cm}^{-1}$ is due to $\mathrm{Cu}-(\mathrm{OH})$ stretching. The peaks that appeared from 2800 to $4000 \mathrm{~cm}^{-1}$ are attributed to $\mathrm{OH}-$ and $\mathrm{C}-\mathrm{O}$ groups on the surface of the $\mathrm{CuO}$ nanoparticles. The peaks that arise at $2927 \mathrm{~cm}^{-1}$ and $2847 \mathrm{~cm}^{-1}$ are due to $\mathrm{C}-\mathrm{O}$ asymmetric stretching. The peaks at $3419 \mathrm{~cm}^{-1}$ denoted $-\mathrm{OH}$ stretching and $1635 \mathrm{~cm}^{-1}$ and $1742 \mathrm{~cm}^{-1}$ denoted $-\mathrm{OH}$ bending [4].

No significant changes seen in FTIR spectra of shock wave treated and untreated $\mathrm{CuO}$ NPs by the influence of shock waves. The reproducibility of the characteristic bends at $622 \mathrm{~cm}^{-1}$ and $513 \mathrm{~cm}^{-1}$ shows the spectacular molecular structure stability of the CuO NPs. Hence, we could confirm that $\mathrm{CuO}$ NPs have higher molecular stability against the impact of shock waves.

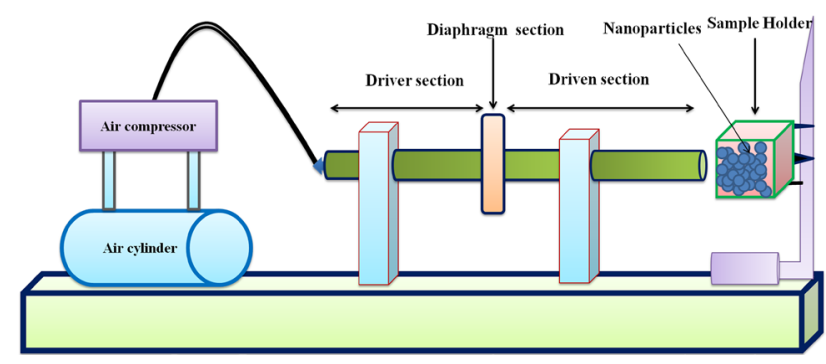

Fig. 1 Schematic diagram of the experimental setup of shock wave loading on the $\mathrm{CuO}$ NPs

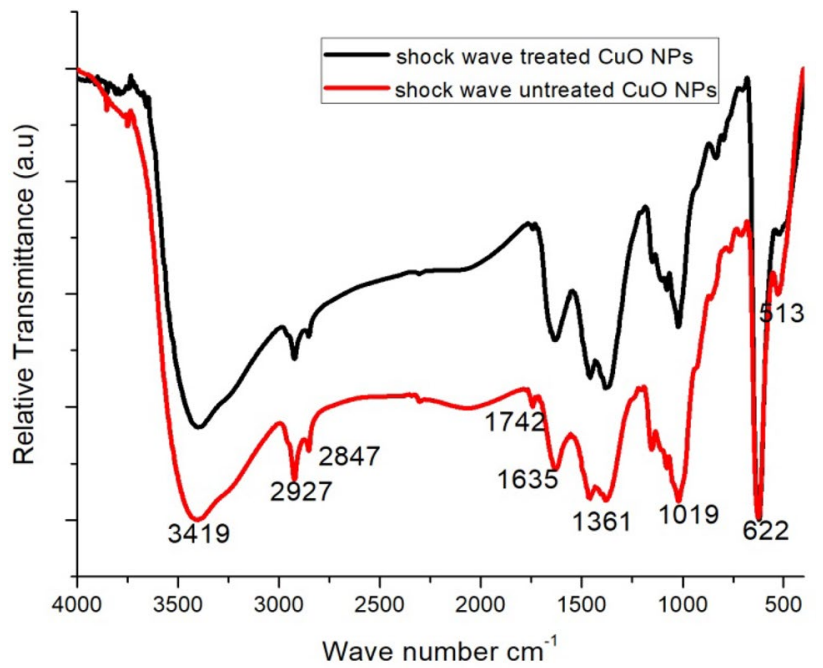

Fig. 2 FTIR spectrum of shock wave treated and untreated copper oxide nanoparticles

\section{Powder X-ray diffraction}

The powder X-ray diffraction is an effective method to explore the structural properties of the crystalline materials and it provides the details about the grain size, structure and phase purity of the materials. The shock wave treated and untreated $\mathrm{CuO}$ NPs were subjected to powder XRD analysis. The obtained powder XRD diffraction patterns of shock wave treated and untreated copper oxide NPs are shown in the Fig. 3. In the case of pre-shock, it is observed that the diffraction peaks occurred at $29.02^{\circ}, 33.65^{\circ}, 43.26^{\circ}, 48.41^{\circ}$, $50.54^{\circ}, 57.54^{\circ}$ and $74.11^{\circ}$ and these lines are indexed as (105), (034), (404), (054), (440), (610), (183) correspondingly which belong to the orthorhombic structure. The obtained peaks are matching well with the JCPDC Card No. 77-1898. The comparison of XRD pattern of shock wave treated and untreated $\mathrm{CuO}$ NPs showed that there is

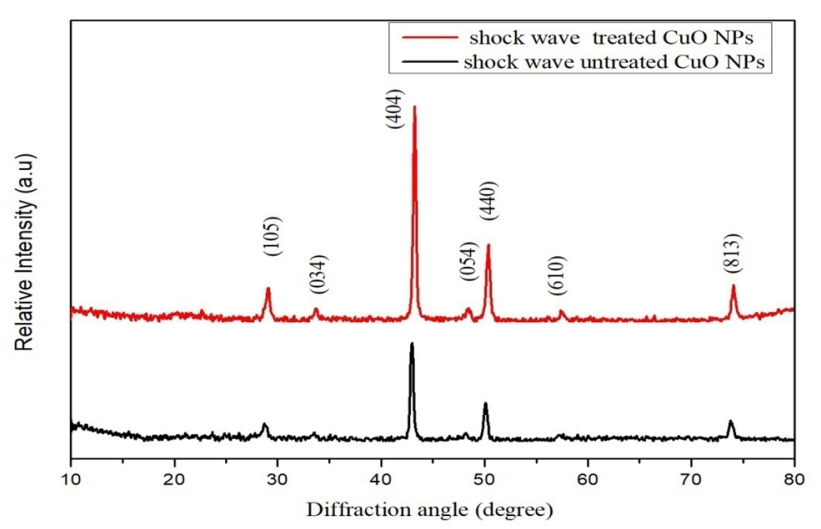

Fig. 3 XRD pattern of shock wave treated and untreated $\mathrm{CuO}$ NPs 
no new peak or a peak shift and not even a peak is smashed after shock wave-loaded conditions. The crystalline phases (planes) may shift from higher to lower angles or vice versa in high-pressure and temperature environments and it is well associated with the nature of the material properties [8]. Interestingly in the present case, there is neither a shift in crystalline phases nor new peaks formation in shock treated $\mathrm{CuO}$ NPs. It exhibits that prepared $\mathrm{CuO}$ NPs have superior structural stability and good resistance of shock waves.

Grain size of shock wave treated and untreated $\mathrm{CuO}$ NPs are determined by using the standard Debye-Scherer formula:

$D=\frac{k \lambda}{\beta \cos \theta}$,

where $D$ denotes the grain size, $K$ refers to a constant, $\lambda$ refers the wavelength of X-ray used for the diffraction study, $\beta$ denotes the full-width half-maximum of the diffraction peaks obtained from the prepared sample and $\theta$ is the angle of the diffraction.

From the powder XRD spectrum, though it is explicitly showed that shock waves did not affect the crystalline structure of the CuO NPs, it is found that the grain size of shock wave treated $\mathrm{CuO}$ NPs have slightly increased from 23 to $25 \mathrm{~nm}$. It may be due to the fusion of very fine grains with the larger grains by the applied shock waves. In general, size of the grains play an indispensable role in the determination of the strength of solid materials whether they are in bulk or nano size [17]. On the other hand, interatomic distance ( $d$-space) is the second key point to determine the structural stability and mechanical strength of materials. In the present case, no changes in the d-space of the system are observed and it explains that shock waves do not affect the grain size as well as structure of the crystalline in prepared $\mathrm{CuO} \mathrm{NPs.}$ If large changes occur in the grain size and inter-atomic distance, it might be greatly affect the structural and mechanical stability of the material. However, the grain size is strained by the external factors (pressure, temperature, etc.) and the $d$-space will be changed. A shock compressive stress is able to change the $d$-spacing into smaller or larger and this effect is well associated with material's structural stability. Structural parameters of the shock wave treated and untreated $\mathrm{CuO}$ NPs are given in Table 1.

Also XRD profile reveals that the applied shock waves do not introduce any deformation, lattice distortion and lattice defects in the $\mathrm{CuO}$ NPs crystalline system. It confirms that $\mathrm{CuO}$ NPs are more stable material as compared to few metal oxide NPs such as $\mathrm{TiO}_{2}, \mathrm{ZrO}_{2}[11,18,19]$. The lattice stress and stress hardening are also calculated from standard Williamson-Hall method [20] and the calculated structural parameters of the shock wave treated and untreated $\mathrm{CuO}$ NPs are listed in Table 1. The insignificant changes in lattice strain and strain hardening due to the plastic deformation of $\mathrm{CuO}$ NPs show the sustainability of the crystalline system of the CuO NPs on loadings of shock waves. The advanced structural stability of the $\mathrm{CuO}$ NPs may be due to the lower bond length and higher ionic bond attraction between $\mathrm{Cu}^{2+}$ and $\mathrm{O}^{2-}$. The results suggest that the prepared $\mathrm{CuO}$ NPs are well appropriate material for extreme surroundings like dynamic high temperature and pressure so as to be used in the aerospace and nuclear reactor applications.

\section{Morphological analysis}

SEM technique is one of the superior methods to investigate the morphology of the NPs. The shock wave treated and untreated samples' surface morphology images are recorded using SEM technique and are shown in the Fig. 4a-d. The shock wave-untreated $\mathrm{CuO}$ NPs are well dispersed, semi-spherical shaped, smooth and unambiguous surface morphology. At the same time the shock wavetreated $\mathrm{CuO}$ NPs are agglomerated and traumatized due to the shock pulses which are evidently shown in Fig. 4c. During the shock wave loading conditions, the materials are also experience to sudden change of other quantities like density, stress, energy and temperature which affect the relative values of mass density and surface area of the particles. But in the present case, though the particles are agglomerated due to shock waves, the particles are having well-resolved boundaries without any morphological changes on the surface of the particles. The average particle sizes of shock wave untreated and treated $\mathrm{CuO}$ NPs are $14.64 \mu \mathrm{m}$ and $17.81 \mu \mathrm{m}$. Thus it shows that there are no considerable changes in the particle sizes of the $\mathrm{CuO}$ NPs. The stability of semi-spherical size of shock wave treated and untreated $\mathrm{CuO}$ NPs proved the firmness of this $\mathrm{CuO}$ NPs.

Table 1 Structural parameters of the shock wave treated and untreated CuO NPs

\begin{tabular}{llllllll}
\hline S. no. & Test conditions & $\begin{array}{l}\text { Predominant peak } \\
(404) \text { position }(2 \theta)\end{array}$ & $\begin{array}{l}\text { Grain size } \\
(\mathrm{nm})\end{array}$ & $\begin{array}{l}\text { Dislocation density } \\
\left(\times 10^{15} \text { lines } / \mathrm{m}^{2}\right)\end{array}$ & $d$-space $(\AA)$ & Lattice strain $(\%)$ & Strain hardening \\
\hline 1 & Untreated CuO NPs & 43.00 & 23 & 1.89 & 2.0868 & 0.0042 & 0.2411 \\
2 & Treated CuO NPs & 43.10 & 25 & 1.60 & 2.0874 & 0.0037 & 0.2123 \\
\hline
\end{tabular}



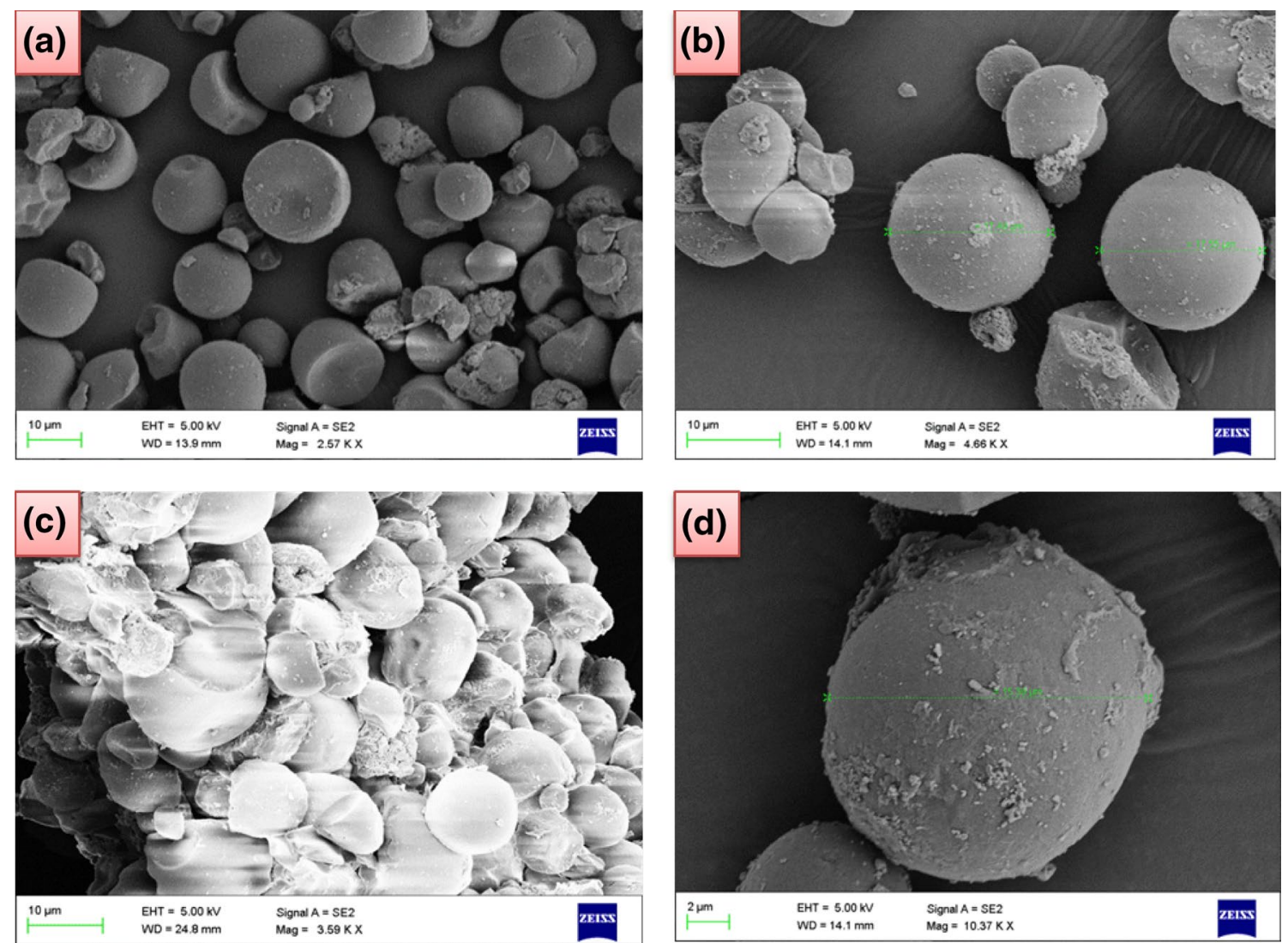

Fig. 4 a-d CuO NPs morphology images of untreated $(\mathbf{a}, \mathbf{b})$ and shock wave treated $(\mathbf{c}, \mathbf{d})$

\section{Conclusion}

Nanoparticles of copper oxide have been effectively synthesized using chemical reduction method and then the synthesized copper oxide nanoparticles were treated with 100 shock pulses having Mach number of 2.4. FTIR spectral analysis confirms the vibration modes of $\mathrm{CuO}$ NPs. The powder XRD analysis confirmed that synthesized $\mathrm{CuO}$ NPs belong to orthorhombic crystalline system and proved its impressive structural and mechanical stability under shock wave-loaded conditions. SEM images clearly show that there is no significant morphological changes occurred in the shock-exposed sample except some negligible differences in surface area of the CuO NPs. Hence, with these results one can conclude that the synthesized $\mathrm{CuO}$ NPs have greater structural stability and highly stable morphology. From these investigations, authors suggest that $\mathrm{CuO}$ NPs can be used in extreme conditions such as high dynamic pressure and temperature, nuclear reactors and aerospace applications.
Open Access This article is distributed under the terms of the Creative Commons Attribution 4.0 International License (http://creativeco mmons.org/licenses/by/4.0/), which permits unrestricted use, distribution, and reproduction in any medium, provided you give appropriate credit to the original author(s) and the source, provide a link to the Creative Commons license, and indicate if changes were made.

\section{References}

1. Usman, M.S., Ibrahim, N.A., Shameli, K., Zainuddin, N., Yunus, W.M.Z.W.: Copper nanoparticles mediated by Chitosan: synthesis and characterization via chemical methods. Molecules 17, 14928-14936 (2012)

2. Veeralekshmi, R., Madhu, K.U.: Magnetic properties of $\mathrm{CuO}-$ CuS nanocomposites. Phys. Appl. Sci. 6, 134-138 (2018)

3. Ethiraj, A.S., Kan, D.J.: Synthesis and characterization of $\mathrm{CuO}$ nanowires by a simple wet chemical method. Nanoscale Res. Lett. 7, 1-5 (2012)

4. Thekkae, V.V., Cernik, P.M.: Green synthesis of copper oxide nanoparticles using gum karaya as a biotemplate and their antibacterial application. Int. J. Nanomed. 8, 889-898 (2013) 
5. Chen, Z., Jiang, S., Sewell, T.D., Gan, Y., Oloriegbe, S.Y., Thompson, D.L.: Effects of copper nanoparticles inclusions on pressure induced fluid-polynano crystalline structural transitions in Krypton. J. Appl. Phys. 116, 233506 (2014)

6. Koteeswara Reddy, N., Jayaram, V., Arunan, E., Kwon, Y.B., Moon, W.J., Reddy, K.P.J.: Investigations on high enthalpy shock wave exposed graphitic carbon nano particles. Diam. Relat. Mater. 35, 53-57 (2013)

7. Devika, M., Koteeswara Reddy, N., Jayaram, V., Reddy, K.P.J.: Sustainability of aligned $\mathrm{ZnO}$ nanorods under dynamic shockwaves. Adv. Mater. Lett. 8, 398-403 (2017)

8. Sivakumar, A., Suresh, S., Anto Predeep, J., Balachandar, S., Martin Britto Dhas, S.A.: Effect of shock waves on dielectric properties of KDP crystal. J. Eletron. Mater. 47, 4831-4839 (2018)

9. Brontvein, O., Jayaram, V., Reddy, K.P.J., Gordon, J.M., Tenne, R.: Two-step synthesis of MoS2 nanotubes using shock waves with lead as growth promoter. Z. Anorg. Allg. Chem. 640, 1115211158 (2014)

10. Vishakantaiah, J., Singh, P., Reddy, K.P.J.: Experimental investigation of nano ceramic material interaction with high enthalpy argon under shock dynamic loading. Appl. Mech. Mater. 83, 66-72 (2011)

11. Jayaram, V., Reddy, K.P.J.: Experimental study of the effect of strong shock heated test gases with zirconia. Adv. Mater. Lett. 7, 100-150 (2016)

12. Jayaram, V., Gupta, A., Reddy, K.P.J.: Investigation of strong shock wave interactions with $\mathrm{CeO}_{2}$ ceramic. J. Adv. Ceram. 3, 297-305 (2014)

13. Branicio, P.S., Zhang, J., Rino, J.P., Nakano, A., Kalia, R.K., Vashishta, P.: Shock-induced microstructural response of monoand nanocrystalline SiC ceramics. J. Appl. Phys. 123, 145902 (2018)
14. Jian, W.R., Li, B., Wang, L., Yao, X.H., Luo, S.N.: Shock response of open-cell nanoporous $\mathrm{Cu}$ foams: effects of porosity and specific surface area. J. Appl. Phys. 118, 165902 (2015)

15. Patil, S.P., Shendye, P., Markert, B.: Molecular dynamics investigation of the shock response of silica aerogels. Materialia 6 , 100315 (2019)

16. Khan, A., Rashid, A., Younas, R., Chong, R.: A chemical reduction approach to the synthesis of copper nanoparticles. Int. Nano Lett. 6, 21-26 (2016)

17. Han, S., Chen, H.-Y., Chu, Y.-B., Shih, H.C.: Phase transformations in copper oxide nanowires. J. Vac. Sci. Technol., B 23, 2557 (2005)

18. Jayaram, V., Singh, P., Reddy, K.P.J.: Study of anatase $\mathrm{TiO}_{2}$ in the presence of $\mathrm{N}_{2}$ under shock dynamic loading in a free piston driven shock tube. Adv. Ceram. Sci Eng. 2, 40-46 (2013)

19. Kalaiarasi, S., Sivakumar, A., Martin Britto Dhas, S.A., Jose, M.: shock wave induced anatase to rutile $\mathrm{TiO}_{2}$ phase transition using pressure driven shock tube. Mater. Lett. 219, 72-75 (2018)

20. Amaya, M., Nakamura, J., Fuketa, T.: Measurements of crystal lattice strain and crystallite size in irradiated $\mathrm{UO}_{2}$ pellet by X-ray diffractometry. J. Nucl. Sci. Technol. 45, 244-250 (2008)

Publisher's Note Springer Nature remains neutral with regard to jurisdictional claims in published maps and institutional affiliations. 